\title{
Fecundity of inbred fruit fly Drosophila melanogaster on different solid culture media: An analysis
}

\author{
Animesh Kumar Mohapatra* \\ Department of Life Science, Regional Institute of Education (NCERT), Bhubaneswar - \\ 751022 (Odisha), INDIA \\ Priyamvada Pandey \\ Department of Life Science, Regional Institute of Education (NCERT), Bhubaneswar - \\ 751022 (Odisha), INDIA \\ ${ }^{*}$ Corresponding author. E-mail: akmncert@gmail.com
}

\begin{abstract}
In the present study, wild-type Drosophila melanogaster collected from stock culture were sub-cultured in three different types of solid culture media (corn, barley and wheat) and control medium for two weeks to produce $F_{1}$ generation. The duration of larval and pupal development, number of pupal cases and hatched flies were scored for first generation. The results were analyzed by using one-way ANOVA, Bonferroni multiple comparison test and paired sample t-test. The control medium showed no pupal cases and hatched flies. Among all the three solid culture media tested, corn meal, barley meal and wheat meal, the latter showed highly significant results at $p \leq 0.001$ than others. However, this parameter was not affected by the carbohydrate amount in the media. The present investigation is an attempt to evaluate the influence of different formulated solid culture media on the life span and reproduction of fruit flies.
\end{abstract}

Keywords: ANOVA, Drosophila melanogaster, Fecundity, Pupa, Solid culture media

\section{INTRODUCTION}

Drosophila melanogaster, commonly known as the fruit fly, is the most extensively used model organism in research due to its high fecundity rate, short life span and adaptation time (Dermerec, 1950; Ranganath, 1999; Ashburner and Roote, 2000; Mitrovski and Hoffman 2001; Markow and O'Grady, 2006; Kenney and Borisy, 2009). The life cycle of the fruit fly has four main stages; egg, larva, pupa, and adult; and duration of its development depends largely on different culturing conditions such as high carbohydrate and protein content, controlled conditions ranging between $20^{\circ} \mathrm{C}$ and $25^{\circ} \mathrm{C}$, yeast for fermentation, absence of predation pressure and optimum $\mathrm{pH}$ range of 3 to 4 (Burnell et al. 1991,Purves et al, 1998; Wayne et al., 2006; Dirkson, 2009; Parvathi et al, 2009; Sandhyarani, 2010). D. melanogaster can be easily bred on overripe and fermented fruits. Earlier studies have shown that different types of media such as corn meal medium, banana jaggery medium, sucrose dextrose medium and maltose corn medium comprising different nutritional value and sugar contents affect the rate of development in fruit fly. Several varieties of yeast and additives including fruits, tomatoes, sugar, raisins, rice, molasses, and oat hulls were

\section{Article Info}

DOI: 10.31018/jans.v10i4.1788

Received: June 6, 2018

Revised: September 17, 2018

Accepted: October 7, 2018

\section{How to Cite}

Mohapatra, A.K. and Pandey, P. (2018). Fecundity of inbred fruit fly Drosophila melanogaster on different solid culture media: An analysis. Journal of Applied and Natural Science, 10(4): $1109-1114$

used for the standardisation of the Drosophila culture media (Baumberger, 1917; Bridges and Darby, 1933; Tatum, 1939; Robertson and Sang, 1944; Jaenike, 1986). The media like agar and cereals like corn flour, oat, wheat etc. are used as the most common ingredients for the solidification and high nutritive supplements for the fruit flyculture media (Spencer, 1943; Flagg, 1998; Tee Sui Yee, 2010).

Past researches have shown that laboratory rearing of these flies is often limited by the longevity and shelf-life of the fruit culture medium used for rearing (Demerec and Kaufmann, 1996). This has prompted researchers to develop an effective solid culture medium supplemented with yeast for laboratory culturing of these flies in large numbers (Wollard et al., 2006). Earlierstudies are primarily dedicated towards understanding the effect of culture medium on the fruit fly development. Little is known about whether the reproduction rate depends on different developmental nutrition in fruit flies. Studies conducted by Widdowson and McCance (1935), Keller (2007) and Lushchak (2012) have revealed that fruits consumed by Drosophila speciesare rich in a mixture of fructose, glucose, sucrose and other carbohydrates; but they are generally not rich in proteins. Taking natural food sources into account, the present 
study has been taken up to develop a suitable culture medium using simple economic methodwhich can results in adequate development as well as increased female fecundity in Drosophila melanogaster.

\section{MATERIALS AND METHODS}

Media preparation: The present study was carried out under Department of Life Science Education at Regional Institute of Education, Bhubaneswar, Odisha. Effectiveness of different solid culture media was studied on the development and fecundity of inbred wild type Drosophila melanogaster. Three types of solid culture media were formulated and plain liquefied agar was used as control medium. Banana culture medium was used as the standard medium for the stock culture due to its higher carbohydrate content as mentioned by Hanschen (1993) and Stocker and Gallant (2007). For the preparation of solid culture media, $100 \mathrm{gm}$ each of three different types of cereals; corn flour, barley and wheat; were used. Each medium was prepared by mixing $250 \mathrm{~mL}$ of distilled water with $40 \mathrm{gm}$ of jaggery and $100 \mathrm{gm}$ of the respective cereal. The media were mixed well, continuously stirred and heated till it thickened. A total amount of $4 \mathrm{gm}$ yeast, $2 \mathrm{~mL}$ of propionic acid $(\mathrm{pH}-2.79)$ followed by $7 \mathrm{gm}$ of agar agar was immediately added to each media with continuous stirring and the final mixtures were allowed to cool for 5 minutes. Each type of culture medium was poured into four sterilised culture vials and few drop of fungal inhibitor, p-hydroxyl benzoic acid methyl ether were added. The culture vials were then plugged with cotton and labelled accordingly. Culturing conditions and transfer of inbred flies: The empty culture vials were autoclaved at $121^{\circ} \mathrm{C}$ for $15-20$ minutes and left for incubation overnight at room temperature. Different media were transferred in to these autoclaved vials separately. The $\mathrm{pH}$ of all the solid culture media was maintained in the range of 3 to 4 . The inbred flies from the stock culture were transferred to an empty bottle. The cotton wool soaked with 4 to 5 drops of $4 \%$ ether was used for plugging the bottle. When all the flies were anesthetised, they were transferred into a petridish within 30 to 40 seconds and examined under microscope. Male and female flies were identified by the presence or absence of sex comb, shape and number of abdominal segments. After identification i, three male flies and three virgin female flies were trans-
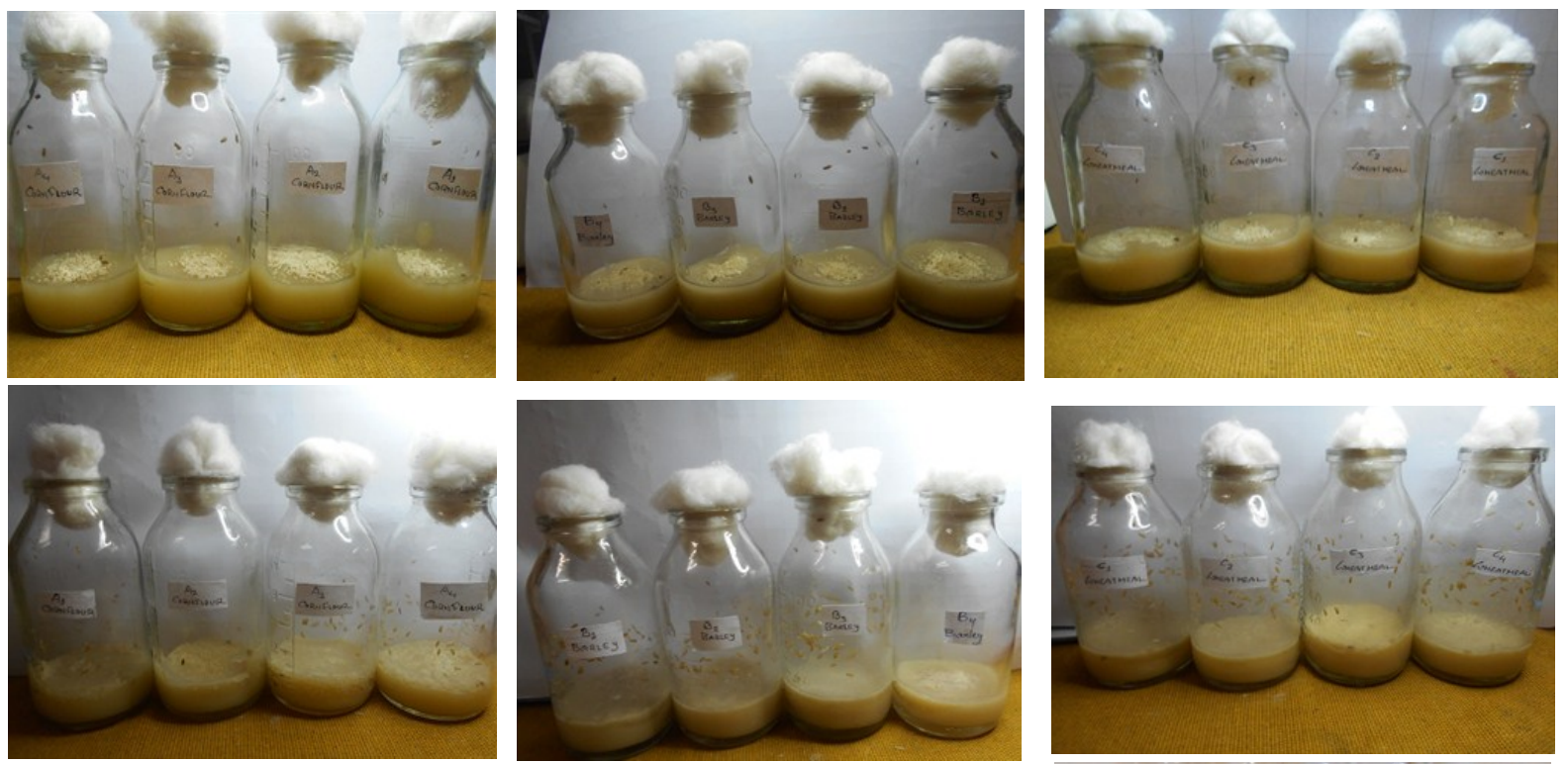

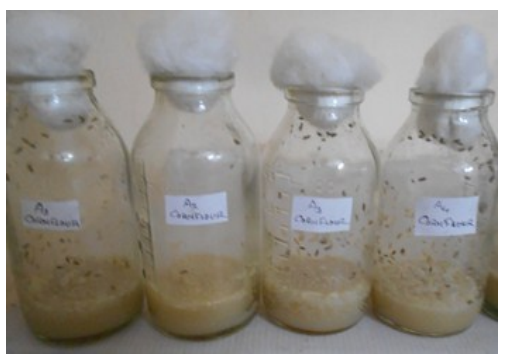

Fig 1. Culture of inbred flies in corn meal solid culture media- $A$ on day 1, day 7 and day 11.

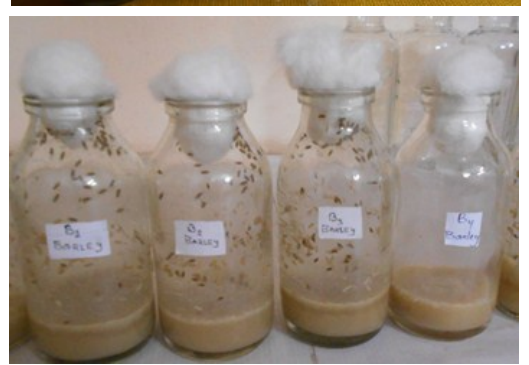

Fig 2. Culture of inbred flies in barley meal solid culture media- $B$ on day 1 , day 7 and day 11 .

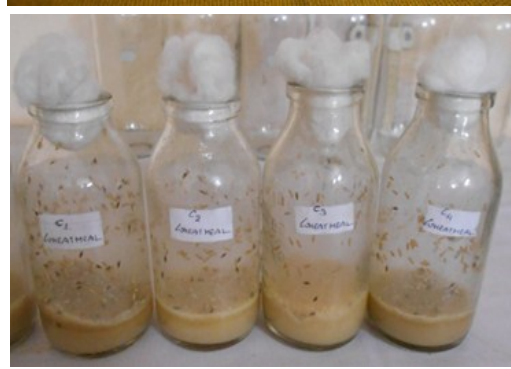

Fig 3. Culture of inbred flies in wheat meal solid culture media-C on day 1, day 7 and day 11. 
Table1. Chemical composition of cereals $(100 \mathrm{gm})$ for solid culture media.

\begin{tabular}{lllllllll}
\hline S.N. & $\begin{array}{l}\text { Cereals } \\
\text { taken }\end{array}$ & $\begin{array}{l}\text { Total carbo- } \\
\text { hydrate } \\
\text { tent } \mathbf{( g m})\end{array}$ & $\begin{array}{l}\text { Dietary } \\
\text { fibre } \\
\mathbf{( g m )}\end{array}$ & $\begin{array}{l}\text { Fat } \\
\mathbf{( g m})\end{array}$ & $\begin{array}{l}\text { Protein } \\
\text { (gm) }\end{array}$ & $\begin{array}{l}\text { Vitamin } \\
\text { (mg) }\end{array}$ & $\begin{array}{l}\text { Trace ele- } \\
\text { ments }(\mathbf{m g})\end{array}$ & $\begin{array}{l}\text { Other con- } \\
\text { stituents }\end{array}$ \\
\hline 1 & Corn flour & 78.7 & 12.2 & 2.8 & 6.8 & - & 281 & - \\
2 & Barley & 77.7 & 15.6 & 1.2 & 9.9 & 5.5 & 613.6 & - \\
3 & Wheat & 72.6 & 12.2 & 1.9 & 13.7 & 8.3 & 938.6 & - \\
\hline
\end{tabular}

Source:"Nutrient data laboratory". USDA.

Table 2. Changes observed in development of $D$. melanogaster among different solid culture media.

\begin{tabular}{llllll}
\hline S.N. & Parameters & Control & Corn meal & Barley meal & Wheat meal \\
\hline 1 & Larval development in days & $0.00 \pm 0.00$ & $6.75 \pm 0.50$ & $7.00 \pm 0.81$ & $7.00 \pm 0.00$ \\
2 & Pupal development in days & $0.00 \pm 0.00$ & $1.50 \pm 1.29$ & $1.50 \pm 0.57$ & $1.00 \pm 0.00$ \\
3 & Number of pupal cases & $0.00 \pm 0.00$ & $53.25 \pm 6.65$ & $76.50 \pm 18.23$ & $129.75 \pm 9.28$ \\
4 & $\%$ Pupal mortality & $0.00 \pm 0.00$ & $4.53 \pm 5.66$ & $18.20 \pm 1.02$ & $28.05 \pm 2.57$ \\
5 & Number of newly emerged $F_{1}$ flies & $0.00 \pm 0.00$ & $50.75 \pm 6.50$ & $62.50 \pm 14.61$ & $93.25 \pm 5.43$ \\
\hline
\end{tabular}

Values represent the Mean \pm SD.

Table 3. One-way ANOVA for the comparison of mean number of pupal cases in first generation of $D$. melanogaster among different solid culture media.

\begin{tabular}{lcccccc}
\hline S.N. & Culture media & Sum of Squares & df & Mean Square & F & Sig. \\
\hline 1 & Between Groups & 34751.250 & 3 & 11583.750 & & \multirow{2}{*}{$000^{* *}$} \\
2 & Within Groups & 1388.500 & 12 & 115.708 & 100.112 & \\
3 & Total & 36139.750 & 15 & & & \\
\hline
\end{tabular}

${ }^{* *}$ significant at $p \leq 0.001$

Table 4. One-way ANOVA for the comparison mean number of hatched flies in first generation of $D$. melanogaster among different solid culture media.

\begin{tabular}{lcccccc}
\hline S.N. & Culture media & Sum of Squares & df & Mean Square & F & Sig. \\
\hline 1 & Between Groups & 18067.250 & 3 & 6022.417 & & \multirow{2}{*}{$000^{* *}$} \\
2 & Within Groups & 856.500 & 12 & 71.375 & 84.377 & \\
3 & Total & 18923.750 & 15 & & & \\
\hline
\end{tabular}

**significant at $p \leq 0.001$

Table 5. Multiple comparison post hoc test (Bonferroni) for the comparison of the number of the pupal cases in the first generation of $D$. melanogasteramong different solid culture media.

\begin{tabular}{llllll}
\hline S.N. & Culture media & Control & Corn meal & Barley meal & Wheat meal \\
\hline 1 & Control & - & $.000^{* *}$ & .000 & $.000^{* * *}$ \\
2 & Corn meal & & - & .060 & $.000^{* *}$ \\
3 & Barley meal & & & - & $.000^{* *}$ \\
4 & Wheat meal & & & & - \\
\hline
\end{tabular}

**significant at $p \leq 0.001$

Table 6. Multiple comparison post hoc test (Bonferroni) for the comparison of the number of the first generation hatched flies of $D$. melanogaster among different solid culture media.

\begin{tabular}{llccll}
\hline S.N. & Culture media & Control & Corn meal & Barley meal & Wheat meal \\
\hline 1 & Control & - & $.000^{* *}$ & $.000^{* *}$ & $.000^{* *}$ \\
2 & Corn meal & & - & .437 & $.000^{* *}$ \\
3 & Barley meal & & & - & $.001^{* *}$ \\
4 & Wheat meal & & & & - \\
\hline
\end{tabular}

**significant at $p \leq 0.001$

Table 7. Paired sample t-tests for the comparison of the number of pupal cases and the number of the first generation hatched flies of $D$. melanogaster among different solid culture media.

\begin{tabular}{llcccccc}
\hline S.N. Culture media & Mean & Std. Deviation & $\begin{array}{c}\text { Std. } \\
\text { Error }\end{array}$ & t & $\begin{array}{c}\text { df } \\
\text { Sig. (2- } \\
\text { tailed) }\end{array}$ \\
\hline 1 & Control pupal cases-hatched flies & - & - & - & - & - & - \\
2 & Corn meal pupal cases - hatched flies & 2.500 & 3.742 & 1.555 & 1.608 & 3 & .206 \\
3 & Barley meal pupal cases - hatched flies & 14.000 & 5.260 & 1.871 & 7.483 & 3 & $.005^{*}$ \\
4 & Wheat meal pupal cases - hatched flies & 36.500 & 5.260 & 2.630 & 13.879 & 3 & $.001^{* *}$ \\
\hline
\end{tabular}

*significant at $p \leq 0.01{ }^{* *}$ significant at $p \leq 0.001$, df-Degree of freedom 
Mohapatra, A.K. and Pandey, P. / J. Appl. \& Nat. Sci. 10 (4): 1109 -1114 (2018)

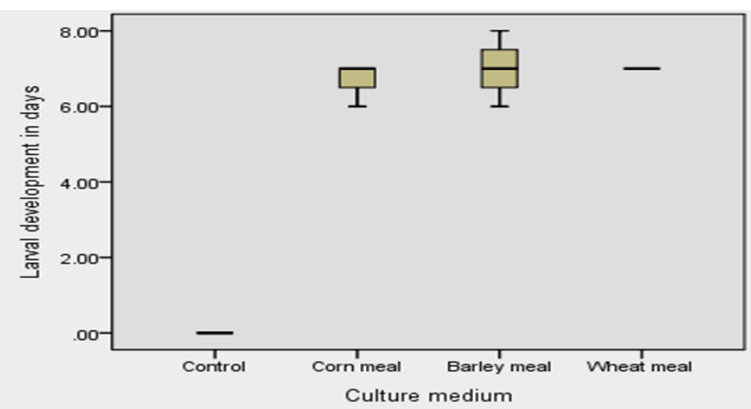

Fig 4. Duration of larval development of D. melanogaster in different solid culture media (in days).

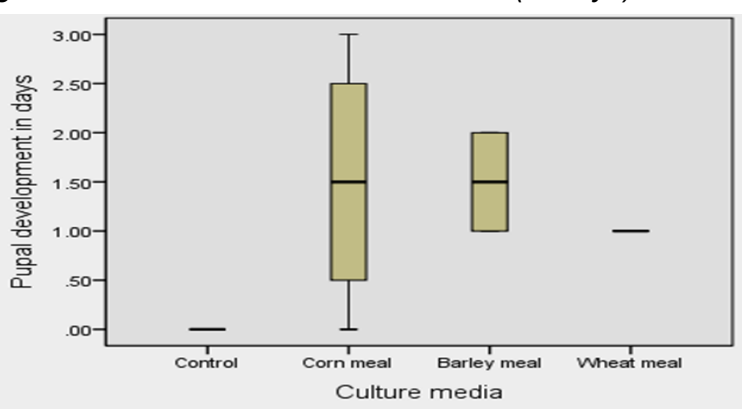

Fig 5. Duration of pupal development of D. melanogaster in different solid culture media (in days).

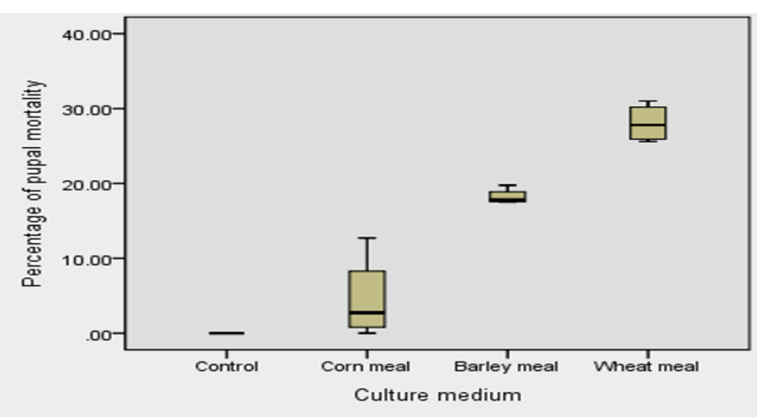

Fig. 6. Percentage of pupal mortality of D. melanogaster in different solid culture media.

ferred into each culture vial which were plugged immediately (Figs. 1-3 ). The media were then incubated in a dark room at $25^{\circ} \mathrm{C}$. The stages of development of the inbred flies in each culture vials were observed every day. When the first generation pupa appeared, the parental flies were removed and after that, the total number of pupal cases in each culture vials were counted everyday till the emergence of adult flies.

Collection of $\mathbf{F}_{\mathbf{1}}$ flies: The first generation flies were counted in each culture vial as soon as they started to emerge. After hatching of all flies in each culture vial, flies were anesthetised and transferred into test tubes containing formalin solution labelled according to the different solid culture media. Flies were transferred to the Petridishes for the total count.

Research design and statistical analysis: In this study, Completely Randomised Design (CRD) was followed and analyses were performed by using SPSS statistical software 20 . The results

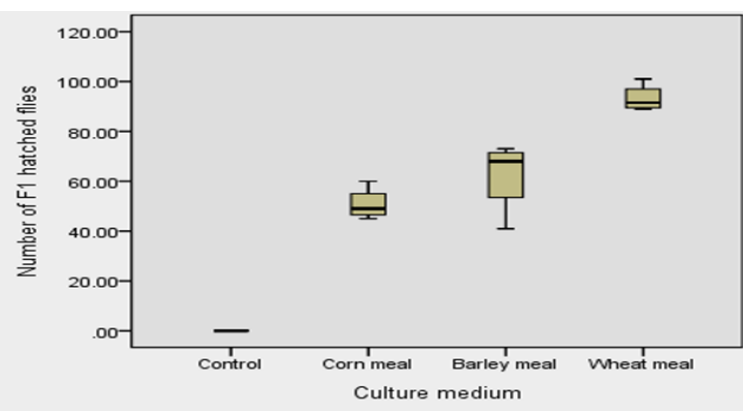

Fig. 7. Number of first generation pupal cases of $D$. melanogaster in different solid culture media.

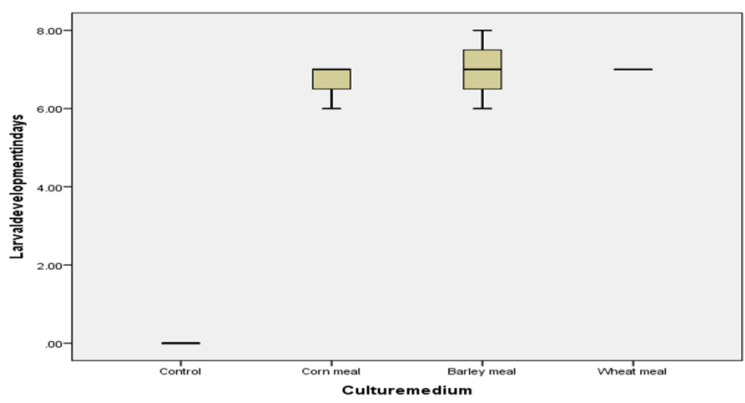

Fig. 8. Number of first generation hatched flies of $D$. melanogaster in different solid culture media.

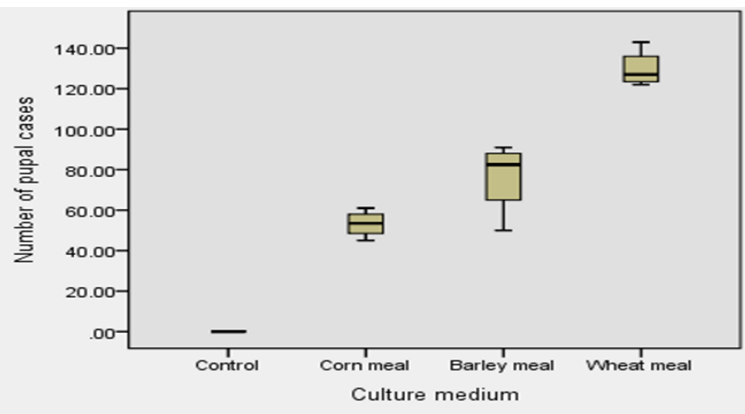

Fig. 9. Number of pupal cases in the first generation of $D$. melanogaster in different solid culture media

were analysed using three different types of tests; one-way Analysis of Variance (ANOVA), Bonferronipost hoc multiple comparison test and paired sample t-test. ANOVA was used to compare the means and variations among and between the results from all types of media. The Bonferronipost hoc multiple comparison tests were performed after ANOVA test to determine the medium having significantly different results compared to other media at significant levelp $\leq 0.001$. The paired sample t-test was conducted to compare the significant differences in the number of pupal cases and hatched flies among all culture media.

\section{RESULTS AND DISCUSSION}

The duration of different developmental stages in days, percentage of pupal mortality, mean number of pupal cases and hatched flies for the first generation in different culture media were analysed (Table 2 and Figs. 4-8 ). The inbred parental flies produced only an average of 53 pupal cases in 
corn meal medium. Rearing of flies on barley meal medium showed moderate results where an average of 76 pupal cases was observed. Wheat meal medium showed the highest number of pupal cases with an average of 129 pupal cases. Similar results were observed for the $F_{1}$ hatched flies. An average of 93 first generation flies hatched in the wheat meal medium followed by 62 flies in barley medium while corn flour medium contained only 50 hatched flies. No pupal cases were observed in the control medium. One-way ANOVA tests for the comparison of population density among all types of solid culture media showed significant differences in the number of pupal cases and hatched flies at $p \leq 0.001$ (Tables 3 and 4 ).

Further, Bonferroni multiple comparison post hoc tests showed that corn flour medium had no significant differences in the number of pupal cases with the barley medium. All other solid culture medium showed significant differences at $p \leq 0.001$ (Table 5). Similar results were observed for the number of first generation hatched flies. All the solid culture medium showed statistically significant results at $p \leq 0.001$ except corn flour medium and barley medium (Table 6). Paired sample ttests for the comparison of number of pupal cases and the number of the first generation hatched flies showed significant differences at $p \leq 0.001$ in wheat medium, at $p \leq 0.01$ in barley medium and no tvalue could be calculated for control medium as the parental flies in this medium failed to survive. These significant differences depict that the number of hatched flies was lower than the number of pupal cases observed. No significant differences was observed for pupal cases and hatched flies in corn flour medium due to less pupal mortality (Table 7).

The fruit flies require an average of 10 days to complete their life cycle if the cultures are maintained at 20 to $25^{\circ} \mathrm{C}$. The eggs are laid within 24 hours, larval and pupal development both takes nearly four days and then there is emergence of new flies. (Geiger, 2002; Gilbert, 2000; Griffiths et al. 2000). The larval development in different formulated solid culture media had taken nearly seven days. The pupal development and emergence of new flies were first reported in wheat meal medium (mean 1 day) followed by corn meal and barley meal medium (mean 1.5 days).In the wheat meal culture medium, the number of pupal cases and $F_{1}$ generation flies was the highest which may be due to high protein content added with carbohydrate in the medium (Table 1). Sang (1962), Fay (1989) and Uysalet al. (2002) in their study reported that the high protein content is vital for the increased reproductive efficiency and regeneration in $D$. melanogaster.

Moreover, maximum pupal mortality was observed in wheat culture media. High pupal mortality was due to the high moisture content and stickiness of the media as a result of which the pupae remain stuck to the media and did not metamor- phose into the adult flies. The other reasons for the increased mortality might be attributed to the autoclaving and fermentation process. Smith (1932) and Skirvin et al. (1986) in their study mentioned that high temperature during sterilization process converts sugar into acidic products and lowers the $\mathrm{pH}$ values of the culture media. Besides, fermentation produces additional acidic products which increase the acidity of the culture medium (Remize et al., 1998).

Further, hydrolysis of agar by acid and lack of nutrients for yeast lowers fermentation and retards the development in fruit fly (Anonymous, 2011). It is observed that corn meal medium showed less pupal mortality. The culture medium containing corn flour remains firm enough to resist liquefaction caused by larval activity (Anonymous, 2007). However, inspite of high carbohydrate content, the number of pupal cases and first generation hatched flies in corn meal medium were found to be very low in comparison to all other media. This might be because of the dryness in the culture media. According to Catchpoole (2005), the desiccation stress is one of the major reasons for the mortality of parent fruit flies, reduced reproductive ability and reduced metamorphosis of the hatched larva in the culture media. In addition, it has been demonstrated by Lushchak et al. (2014) that the media containing high carbohydrate especially sucrose and low protein content may lower egglaying capability of fruit flies on the diets. In the liquefied agar control medium, the fruit flies were not able to survive as it lacks nutritional values which are essential for growth, reproduction and the development of flies.

\section{Conclusion}

On the basis of the findings, the number of first generation pupal cases and hatched flies observed in wheat meal culture medium was significantly higher than the results for all other culture media. Hence, it can be inferred that the wheat meal medium is the most suitable solid culture medium for breeding $D$. melanogaster. Conversely, in the control medium fruit flies failed to survive and breed.

\section{ACKNOWLEDGEMENTS}

The authors are grateful to Prof. P. C. Agarwal, Principal and Dr. Ramakant Mohalik, Associate Professor, Regional Institute of Education, Bhubaneswar for extending their valuable suggestions for statistical analysis of the data.

\section{REFERENCES}

1. Anonymous (2007). Basic Methods of Culturing Drosophila. http://flystocks.bio.indiana.edu/Fly_Work/ culturing.html.

2. Anonymous (2011).Genetics.http://www.indiana.edu/ oso/lessons/Genetics/bw_st.html\#top.

3. Ashburner, M. and Roote, J. (2000). Laboratory cul- 
ture of Drosophila. In: Drosophila protocols, ed. W. Sullivan, M. Ashburner, and R. S. Hawley, Cold Spring Harbor Laboratory Press, Cold Spring Harbor,NY, pp 585-599.

4. Baumberger, J. (1917). Solid media for rearing Drosophila. The American Naturalist, 51: 447-448.

5. Bridges, C. and Darby, H. (1993). Culture media for Drosophila and the $\mathrm{pH}$ of media. TheAmerican Naturalist, 67: 437-472.

6. Burnell, A. N., Reaper, C. and Doherty, J. (1991). The Effect of Acclimation Temperature on Enzyme Activity in Drosophila melanogaster. Comparative Biochemistry and Physiology Part B: Comparative Biochemistry, 98 (4): 609-614.

7. Catchpoole, D. 2005. Too dry for a fly. Creation, 28 (1):34-35

8. Dermerec, M. (1950). Biology of Drosophila. New York: John Wiley and Sons, Inc,pp 632.

9. Demerec, M. And Kaufman, B.P. (1996). Drosophila guide: introductiont to the genetics and Cytology of Drosophila melanogaster.10thed. CarnegielnstituteofWashington, USA, pp 1-27.

10.Dirkson, R. (2009). A Drosophila Protocol for Teaching Homeobox Genetics and Homeotics Mutations. http:// www.slideshare.net/RDirksen/drosophila-protocol.

11.Fay, H.A.C. (1989). Multi-host species of fruit fly. In: World Crop Pest. Fruit flies, their biology, natural enemies and control. Edited by Robinson A.S. and Hooper G. Ed. Elsevier, New York,3b:129-138.

12.Flagg, R. O. (1998). CarolinaTM Drosophila Manual. Carolina Biological Supply Company, Burlington, NC, pp 1-23.

13.Geiger, P. (2002). An Introduction to Drosophila melanogaster. http://biology.arizona.edu/sciconn/lessons2/ geiger/intro.html.

14.Gilbert, S. F. (2000). Developmental Biology.6th ed. Sunderland: Sinauer Associates, pp 294-296.

15.Griffiths, A. J., Miller, J. H., Suzuki, D. T., Lewontin, R. C. and Gelbart, W. M. (2000). sex chromosomes and sex-linked inheritance. An Introduction to Genetic Analysis 7th ed. W. H. Freeman. New York.

16. Henschen, F. (1933). The Nobel Prize in Physiology or Medicine,1933 Thomas $\mathrm{H}$. MorganAwardCeremonySpeech.https://www.nobelprize.org/ nobel_prizes/medicine/laureates/1933/.

17.Jaenike, J. (1986). Feeding behavior of future fecundity in Drosophila. The American Naturalist, 127:118-123

18.Keller, A . 2007. Drosophila melanogaster's history as a human commensal. Curr Biol17: 77 -81.

19.Kenney, D. E. and Borisy, G. G. 2009. Thomas Hunt Morgan at the Marine Biological Laboratory: Naturalist and Experimentalist. Genetics, 181(3):841-846.

20.Lushchak, O.V., Gospodaryov, D.V ., Rovenko, B.M . (2012). Balance between macronutrients affects life span and functional senescence in fruit fly Drosophila melanogaster. J Gerontol, 67:118-125.

21.Lushchak, O.V., Gospodaryov, D.V., Rovenko, B.M., Yurkeyych, J.S., Perkhulyn, N.V. and Lushchak, V.I., (2014). Specific dietary carbohydrates differentially influence the life span and fecundity of Drosophila melanogaster. J Gerontol., 69 (1): 3-12.

22.Markow, T. A. and O'Grady, P. M. 2006. Distribution in Drosophila. A Guide to Species Identification and Use. California, Elsevier.

23.Mitrovski, P. and Hoffman, A. A. 2001. Postponed Reproduction as an Adaptation to Winter Conditions in Drosophila melanogaster. Evidence for Clinical Varia- tion under Semi-Natural Conditions. Proceedings: Biological Sciences, 268(1481):2163-2168.

24.Parvathi, D., Amritha, A. and Paul, S.F.D. 2009. Wonder animal model for genetics-Drosophila melanogaster Its life cycle and breeding methods- A review. Sri Ramachandra Journal of Medicine, 2(2):33-38

25.Purves, W. K., Orians, G. H., Heller, H. C. and Sadava, D. 1998. Life: The Science of Biology. Sinauer Associates. Sunderland.

26.Ranganath, H. A. (1999). Teaching and learning genetics with Drosophila. http://www.ias.ac.in/resonance/ Feb1999/pdf/Feb1999Classroom.pdf.

27.Remize, F., Roustan, J. L., Sablayrolles, J. M., Barre, P. and Dequin, S. (1998). Glycerol Overproduction by Engineered Saccharomyces cerevisiae Wine Yeast Strains Leads to Substantial Changes in By-Product Formation and to a Stimulation of Fermentation Rate in Stationary Phase. Applied and Environmental Microbiology, 65(1): 143-149.

28.Robertson, F., and Sang, J. (1944). Theecological determinants of population growth ina Drosophila culture. I. Fecundity of adult fruitflies. Proceedings of the Royal Society of London. Series B, Biological Sciences, 132:258-277.

29.Sandhyarani, N. (2010). Drosophila melanogaster Life Cycle. http://www.buzzle.com/articles/drosophilamelanogaster-life-cycle.html.

30.Sang, J. H. (1956). The Quantitative Nutritional Requirements of Drosophila melanogaster. Journal of Experimental Biology, 33:45-72.

31.Skirvin, R. M., Chu, M. C., Mann, M. L., Young, H., Sullivan, J. and Fermanian, T. (1986). Stability of tissue culture medium $\mathrm{pH}$ as a function of autoclaving, time, and cultured plant material. Plant Cell Reports, 5: 292-294.

32.Smith, M. L. (1932). The effect of heat on sugar solutions used for culture media. Biochem J., 26(5):14671472.

33.Spencer, W. P. (1943). Drosophila culture with a minimum of agar. The Ohio Journal of Science, 43 (4):174-175.

34.Stocker, H. and Gallant, P. (2007). Getting Started: An Overview on Raising and Using Drosophila. In Drosophila: Methods and Protocols. New York: Humana Press Inc.

35.Tatum, E.(1939). Nutritional requirements ofDrosophila melanogaster. Proceedings of the

36.National Academy of Science of the United States of America, 25: 490-497.

37.Tee Sui Yee. (2010). Optimization of fruit fly (Drosophila melanogaster) Culture media for higher yield of offspring. Universiti Tunku Abdul Rahman, pp 1-57.

38.Uysal, H., Aydogan, M. N. and Algur, O. F. 2002. Effect of single cell protein as a protein source in Drosophila Culture. Brazilian Journal of Microbiology, 33: 314-317.

39.Wayne, M. L., Soundararajan, U. and Harshman, L. G. (2006). Environmental stress and reproduction in Drosophila melanogaster: starvation resistance, ovariole numbers and early age egg production. EMC Evolutionary Biology, 6:57.

40.Widdowson, E. M.and McCance, R.A. (1935).The available carbohydrate of fruits:determination of glucose, fructose, sucrose and starch, Biochem J., $129: 151-156$.

41.Wollard, L., Klein B., Carlson, J.D. and Carlson, K.A. (2006). Rearing media as a variable in fruit fly fecundity: An activity to introduce scientific methods of inquiry to biology students. Bioscene, 32(3):24-29. 